Pensamiento Crítico N. ${ }^{\circ} 15$, pp. 45-60

\title{
Determinantes de la violencia familiar contra las mujeres en el Perú: una estimación econométrica ${ }^{1}$
}

\author{
Juan León Mendoza
}

\section{RESUMEN}

El presente artículo tiene como objetivo fundamental analizar el problema de la violencia física, psicológica y sexual contra las mujeres por parte de sus cónyuges.

Con la ayuda de un modelo microeconómico formulado y utilizando la información proporcionada por ENDES 2009, los resultados de la regresión econométrica logística indican que las principales variables subyacentes que determinan la probabilidad de generación de acciones de violencia conyugal contra las mujeres en el Perú son las siguientes: (a) que la mujer esté desempleada o no inmersa en el mercado laboral, (b) que haya sufrido agresiones físicas por parte de sus padres durante su infancia o niñez y, (c) que esté en situación de casada o de convivencia.

Palabras clave: Violencia conyugal, violencia física, violencia psicológica, violencia sexual.

1 Primer lugar en el concurso de proyectos de investigación, categoría violencia, del "Programa de Proyectos Multidisciplinarios de Investigación 2010", organizado por el Vicerrectorado de Investigación de la Universidad Nacional Mayor de San Marcos. Colaboraron en la investigación: Richard Roca Garay, Ciro Calero Castillo, Blanca Reyna Marín y Raúl León Caparó. 


\title{
Pensamiento Crítico N. 15
}

\begin{abstract}
This article aims at fundamental to analyze the problem of physical, psychological and sexual violence against women by their spouses.

With the aid of a microeconomic model formulated and using the information from ENDES 2009, the results of logistical regression econometric show that the main underlying variables that determine the probability of generating actions of spousal violence against women in Peru are: (a) that the woman is unemployed or not immersed in the labor market, (b) she has been physically assaulted by their parents during infancy or childhood, and (c) she is in a situation of married or conviviality.
\end{abstract}

Keywords: violence, physical violence, psychological violence, sexual violence.

\section{Antecedentes empíricos}

Existen tres tipos de violencia doméstica o conyugal: violencia física, psicológica y sexual. A nivel internacional, los trabajos de investigación citan frecuentemente, como factores asociados a la violencia doméstica, al nivel socioeconómico del hogar, al nivel de educación, al estado civil, la edad, la experiencia infantil de agresión por parte de los padres, la ocupación de la mujer y de su pareja, el número de hijos, la brecha o asimetría de poder, el consumo de alcohol, el número de años de unión (Castro y Riquer, 2003).

El nivel educativo y el estar empleada se convierten en factores que tienden a "proteger" a las mujeres de la violencia de sus parejas: a mayor nivel de educación alcanzada y mayor nivel de ocupación laboral, menor es la probabilidad de que sea sujeto de violencia (Ackerson, 2008). Por otro lado, Ruiz-Pérez (2006) encuentra que la probabilidad de la violencia es mayor cuando la mujer está separada de la pareja, la mujer sea más joven y cuando tenga más de tres hijos menores en la familia.

En el Perú, un primer trabajo de corte econométrico fue ejecutado por González de Olarte y Gavilano (1999), pero sólo para Lima Metropolitana. Dicha investigación encuentra que, la probabilidad de que la mujer sufra violencia física es mayor en la medida en que: la pareja tenga trabajo, que se encuentre en estado de convivencia o separada, el barrio en que radica sea violento y, que la mujer pertenezca a alguna red social. La variable pobreza no muestra significancia estadística. 


\section{Juan León Mendoza}

Por otro lado, la probabilidad de sufrir violencia psicológica es mayor en tanto que: la pareja esté trabajando, cuando la familia viva en estado de pobreza y, cuando la mujer pertenezca a una red u organización social. La violencia sexual está condicionada, en sentido directo, por la situación de pobreza de la familia, la ausencia de embarazo en la mujer, la pertenencia de la mujer a una red social y, cuando más joven sea ella. Se debe destacar el hallazgo de que las variables que determinan la violencia física no necesariamente determinan la violencia psicológica o sexual y viceversa.

Matos y Sulmont (2009) es el único que hizo uso de la técnica econométrica logística y trabajó con la data de la Encuesta Demográfica y de Salud Familiar (ENDES). Con información de ENDES 2007, encuentra que la ocurrencia de la violencia conyugal contra la mujer está asociada principalmente a los indicadores del patriarcalismo y el control machista. Específicamente, el consumo recurrente del alcohol es uno de los factores que incrementa la probabilidad de ocurrencia de dicho fenómeno.

Esta investigación si bien efectúa regresiones para los tres tipos de violencia conyugal (violencia física, psicológica y sexual), no se fundamenta en un modelo o teoría micro que tome en cuenta la racionalidad de los involucrados en general o del agresor masculino en particular.

\section{El modelo}

Tomando en cuenta los aspectos señalados en el capítulo de antecedentes empíricos, a continuación, formulamos un modelo microeconómico que trata de teorizar la violencia del varón contra su cónyuge femenino.

El modelo supone que el varón es un agente maximizador de utilidad (felicidad o bienestar) individual. Por ello, cuando decide tener una pareja de sexo femenino trata de alcanzar el máximo nivel de bienestar en la vida. Además, se asume que la sociedad es predominantemente machista y patriarcal. En este contexto, el varón visualiza a la mujer como un bien que le brinda una serie de servicios (compañía, apoyo, sexo, amor, procreación y crianza de hijos, etc.). Además éste conceptúa a la pareja femenina como de "su propiedad" y trata de tenerla bajo su dominio o control. 


\section{Pensamiento Crítico N. 15}

Dado los supuestos, la función de utilidad a maximizar (U) depende de los servicios que brinda la mujer a su pareja (S) y de la violencia conyugal que aplica el varón (V):

$$
\mathrm{U}=\mathrm{U}(\mathrm{S}, \mathrm{V})
$$

El servicio femenino es un "bien", es decir, una mayor cantidad de servicios femeninos le genera un mayor nivel de satisfacción, felicidad o bienestar a la pareja conyugal. En tanto que la violencia es un "mal". La violencia per se aplicada a su pareja, no ocasiona placer o felicidad al varón; este hecho de alguna manera le ocasiona cierto sentimiento de culpa, malestar, disgusto o preocupación. Por ello se asume que: Us $>0$, Uss $<0, \mathrm{Uv}<0$

Us $>0$ y Uss $<0$ indica que la utilidad marginal que reporta el servicio femenino a su pareja es positiva, pero decreciente. $\mathrm{Uv}<0$ indica que la utilidad marginal que genera la violencia es negativa, es decir, la violencia más que utilidad genera una desutilidad.

En el Gráfico 1, en el plano de la cantidad de servicios femeninos brindados a la pareja (S) y el nivel de violencia masculina a su cónyuge (V), la función de utilidad se representa mediante las llamadas curvas de indiferencia de pendiente positiva. Cada curva de indiferencia $U$ refleja un nivel de utilidad. El individuo masculino aspira a obtener o disfrutar de un mayor nivel servicios femeninos con un mismo grado de violencia o control a su pareja, por ello, éste prefiere a $U_{2}$ sobre $U_{1}$ o sobre $U_{0}$.

La búsqueda de maximización de utilidad, es decir, acceder a los servicios femeninos, está sujeta a ciertas restricciones o costos.

La unión o el establecimiento de una relación conyugal implica una relativa concesión o pérdida de la independencia personal; es decir, aceptar cierto grado de control o sometimiento por parte de la pareja. En este marco, el control que aplica el varón a su pareja ya refleja cierto grado de violencia (violencia psicológica). Por ello, el varón, cuando trata de acceder a un mayor nivel de servicios de su cónyuge tiende a aplicar mayor nivel de control o de violencia. 


\section{Juan León Mendoza}

Sin embargo, la generación de la violencia (V) no sólo está condicionada -en sentido directo- por el deseo del varón de obtener a un mayor nivel de servicio de su pareja (S), sino también por el grado de machismo (GM) y de patriarcalismo (GP) reinante en la sociedad. El machismo y el patriarcalismo se resumen en el grado de dominio del varón hacia la mujer (GD). A mayor grado de dominio, se produce un mayor grado de control o violencia. Lo manifestado queda resumido en la llamada ecuación de violencia:

$$
\mathrm{V}=\mathrm{V}(\mathrm{S}, \mathrm{GM}, \mathrm{GP})=\mathrm{V}(\mathrm{S}, \mathrm{GD})
$$

El grado de dominio está relacionado a la capacidad de someter a la pareja. En una familia no democrática, en el proceso de la convivencia, el fuerte o el que tiene más poder relativo somete al débil.

El poder del varón está compuesto básicamente por el poder económico relativo (PER), el poder social relativo (PSR) y el poder de conocimiento o información relativo (PCR). Estos tipos de poder determinan el grado de dominio dentro de la familia:

$$
\mathrm{GD}=\mathrm{GD}(\mathrm{PER}, \mathrm{PSR}, \mathrm{PCR})
$$

El poder económico relativo se define como el poder económico del varón (PEv) menos el poder económico de la mujer $(\mathrm{PEm})$, es decir, PER $=\mathrm{PEv}-\mathrm{PEm}$. En el mismo sentido, el poder social relativo es igual al poder social del varón (PSv) menos el poder social de la mujer (PSm). Formalmente: PSR $=\mathrm{PSv}-\mathrm{PSm}$. El poder de conocimiento relativo es igual al poder de conocimiento del varón (PCv) menos el poder de conocimiento de la mujer $(\mathrm{PCm}): \mathrm{PCR}=\mathrm{PCv}-\mathrm{PCm}$.

El poder económico está determinado por la capacidad económica, es decir, por la capacidad de generar ingresos para la familia. Asumiendo que la principal fuente de ingresos es el empleo en el mercado laboral, si el varón está empleado (Lv), éste tendrá un mayor poder relativo dentro de la familia. Sin embargo, si la mujer está empleada (Lm) o que ocupa un puesto de mayor calificación en el mercado laboral, el poder económico relativo del varón tenderá a disminuir. 


\section{Pensamiento Crítico N. ${ }^{\circ}$}

Si el varón está empleado y la mujer está desempleada o no participa en el mercado laboral, la pareja masculina gozará de un mayor poder económico relativo, por ende, mayor capacidad de dominio o de patriarcado, lo que dará pie a la generación de un mayor nivel de violencia conyugal contra la mujer.

El poder social tiene que ver con el capital social, es decir, con el tejido de relaciones y contactos personales o institucionales con que cuenta la persona. Si el varón está más integrado a la sociedad o que la mujer está relativamente aislada del mismo, entonces, la pareja masculina gozará de un mayor poder social relativo, lo cual se traducirá en la generación de una mayor violencia conyugal, dado que se acentúa el grado de dominio machista y patriarcal.

El poder de conocimiento e información está relacionado al capital humano de la persona, es decir, al stock de conocimientos acumulados. A su vez, la acumulación del capital humano depende básicamente de los aspectos de la educación. Una persona que alcanza un mayor nivel de educación, posee un mayor nivel de conocimientos y está mejor informado. Por ello, el poder de conocimiento relativo estará determinado por el nivel de educación alcanzado por el varón (Ev) respecto al de la mujer (Em).

El nivel de educación del varón condiciona la generación de dominio de información y por ende violencia conyugal mediante dos canales contrapuestos. Por un lado, un mayor nivel de educación de éste puede generar mayor dominio de conocimiento $e$ información de manera en que pueda cometer actos de abuso (violencia). Por otro lado, un varón mejor informado, puede ser más respetuoso de las reglas de convivencia cooperativa y de los derechos humanos de la mujer, por lo que se podría generar menores niveles de violencia.

Dado el nivel de educación del varón, el hecho de que la mujer alcance un mayor nivel de educación reduce el poder relativo de conocimiento y de dominio del cónyuge varón. Una mujer, cuanto más educada o instruida es, hace valer y respetar mucho más sus derechos, por ende, permite menos actos de violencia o de agresión por parte de su pareja. Adicionalmente, en tanto que la educación incrementa la productividad y competitividad personal, una mujer con mayor educación puede generar un mayor nivel de ingreso económico, lo cual, en la medida en que reduce el poder económico relativo del varón, tiende a coadyuvar a la reducción de la violencia conyugal contra la mujer. 


\section{Juan León Mendoza}

Efectuando los reemplazos correspondientes, la ecuación de violencia -a la que está sujeta la maximización de utilidad-queda especificada de la siguiente manera:

$$
\mathrm{V}=\mathrm{V}(\mathrm{S}, \mathrm{GD})=\mathrm{V}(\mathrm{S}, \mathrm{Lv}, \mathrm{Lm}, \mathrm{PSv}, \mathrm{PSm}, \mathrm{Ev}, \mathrm{Em})
$$

En el plano de servicios de la mujer (S) y la violencia (V), la lógica de la ecuación de violencia puede ser representado gráficamente mediante una curva de pendiente positiva (Véase Gráfico 1). Dicha curva podemos denominarla como la "curva de la violencia" $\left(\mathrm{CV}_{0}\right)$.

La curva de violencia tiene las siguientes propiedades: $V_{s}>0$, Vss $>0$. Es decir, el acceso a mayores niveles de servicios femeninos genera mayor nivel de violencia conyugal por parte del varón, pero a costos crecientes (en términos de violencia).

Con la finalidad de mostrar cómo se determina el nivel de violencia conyugal y por qué éste puede agudizarse o incrementarse, veamos gráficamente el escenario de dos tipos de régimen familiar. Primero, el caso de una familia plenamente democrática $y$, segundo, el caso de una familia machista y patriarcal.

En el Gráfico 1, en el caso donde existe plena democracia conyugal (están ausentes los actos de machismo y el patriarcalismo), el intercepto de la curva de violencia estará localizado en el origen de los ejes S y V; ello, debido a que la ecuación de violencia se reduce a $\mathrm{V}=\mathrm{V}(\mathrm{S})$.

El varón maximiza utilidad en el punto A. En ella, la curva de indiferencia $\left(\mathrm{U}_{1}\right)$ es tangente a la curva de violencia $\left(\mathrm{CV}_{0}\right)$. Al punto de equilibrio maximizador le corresponde un nivel de violencia de $\mathrm{V}_{0}$.

El modelo indica que, en una unión conyugal, por más que las decisiones familiares se tomen democráticamente o que exista un empoderamiento equitativo, habrá necesariamente cierto nivel de control, por ende, de violencia.

En el entendido de que una unión o relación conyugal implica cierto respeto o pertenencia mutua de pareja, es decir, en la medida en que la pareja es "su" pareja, 


\section{Pensamiento Crítico N. 15}

racionalmente el individuo (la mujer o el varón) aplicará un control relativo sobre su cónyuge. Una persona con pareja ya no es "totalmente libre", se pierde la libertad absoluta; la libertad o la independencia personal se torna parcial. Establecer una relación conyugal implica asumir responsabilidades, es decir, deberes, pero, también derechos. El derecho de uno sobre otro, en tanto que recorta la libertad absoluta, ya implica cierto grado de control.

Entonces, $\mathrm{V}_{0}$ es un nivel de control óptimo o mínimo; una especie de violencia mínima que cuenta con la aceptación voluntaria de la mujer. Equivale al costo (pérdida de privacidad absoluta) que asume la mujer por establecer una relación conyugal. Es un nivel de violencia con el que el varón está de acuerdo, concordante con que éste también esté aceptando incurrir en un costo de pérdida de independencia absoluta.

En un segundo escenario, la introducción de aspectos o variables relacionadas al machismo y patriarcado implica el traslado de la curva de violencia de $\mathrm{CV}_{0}$ a $\mathrm{CV}_{1}$. Ahora el punto de tangencia entre la curva de indiferencia y la curva de violencia se localiza en B. En ella, el cónyuge varón también maximiza utilidad.

En relación a la situación de un régimen familiar democrático, la presencia de factores relacionados al machismo y patriarcado incrementa el nivel de la violencia conyugal de $\mathrm{V}_{0}$ a $\mathrm{V}_{1}$. En otros términos, según el modelo, el hecho de que la mujer no esté empleada, alcance menores de educación, no esté relativamente integrado a una red social o que la pareja varón tenga menores niveles de educación, tenga empleo y que cuente relativamente con más relaciones sociales, genera un mayor grado de dominio del varón tal que tiende a incrementarse el nivel de la violencia conyugal contra la pareja.

La simulación gráfica muestra que las actitudes machistas y patriarcales no sólo generan mayores niveles de violencia conyugal, sino que también disminuye el nivel de servicios efectivos que recibe el varón de parte de su pareja, es decir, el varón accede a una menor cantidad de servicios femeninos. En el Gráfico 1, el nivel de servicios que brinda la mujer -a su pareja- disminuye de $S_{0}$ a $S_{1}$. 


\section{Juan León Mendoza}

Gráfico 1: Optimización en una familia democrática y patriarcal/machista

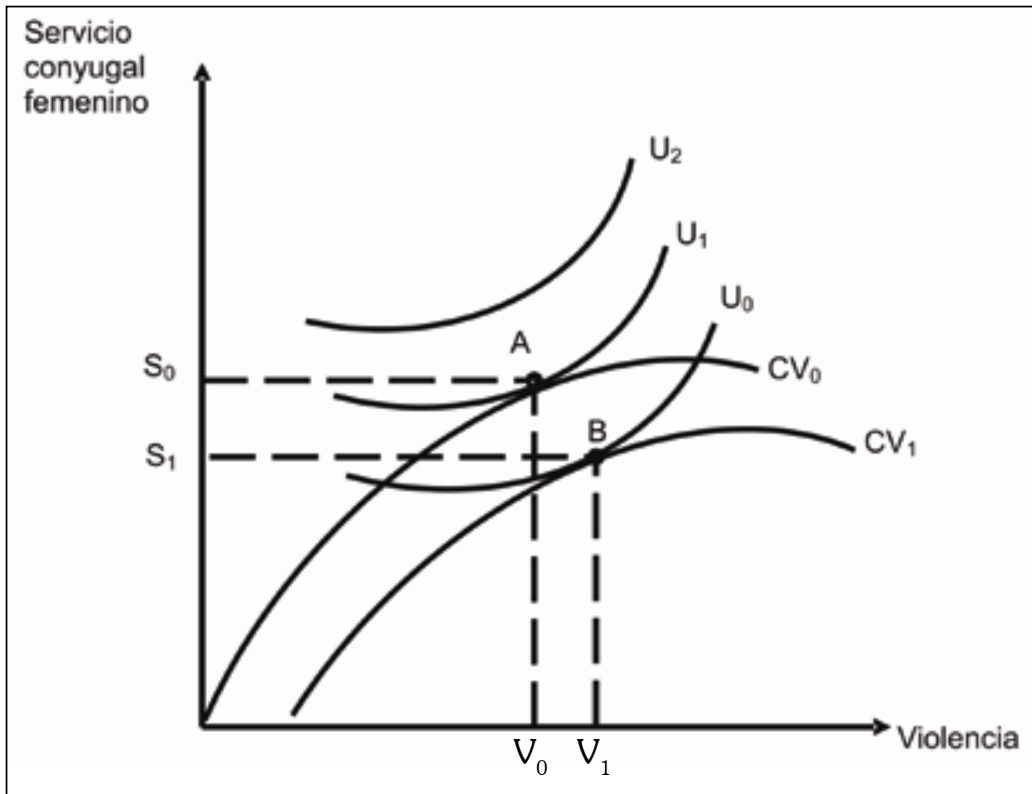

El resultado de esta simulación no es de sorprender y tiene mucha lógica. Generalmente a ninguna persona le agrada que le sometan, controlen o que le violenten en sus derechos. Por ello, es de esperar que cuando se incrementa el nivel de la violencia conyugal hacia la mujer, ella tiende a reducir la cantidad y la calidad de sus servicios hacia su pareja (amor, cariño, ayuda, compañía, fidelidad).

Finalmente, el modelo indica que una familia democrática genera mayores niveles de bienestar a la pareja masculina. Según el Gráfico 1, el punto óptimo A -que corresponde a un régimen democrático- permite alcanzar una utilidad igual a $U_{1}$, el mismo que es mayor que el punto $\mathrm{B}$ que corresponde al régimen machista y patriarcal. Si el varón cede en su grado de dominio o control machista, la mujer responderá elevando la cantidad y calidad de trato a su pareja, lo cual obviamente será de mayor agrado o satisfacción para éste. 


\section{Pensamiento Crítico N. 15}

El modelo formulado permite plantear la hipótesis de que la probabilidad de una violencia física, psicológica y sexual contra la mujer está asociada a factores educativos, de empleo, y de aspectos socioeconómicos pertenecientes al hogar o familia.

\section{Evidencia econométrica}

Con la finalidad de evaluar la hipótesis general formulada, efectuamos regresiones econométricas para cada tipo de violencia (física, sexual y psicológica) utilizando el modelo probit. Las variables explicativas consideradas, corresponden a las contenidas en el modelo teórico que elaboramos. Además, en ese marco se agregan variables incorporados en otros estudios empíricos. Los datos utilizados provienen de LA ENCUESTA DEMOGRÁFICA Y DE SALUD FAMILIAR- ENDES 2009, ejecutado a nivel de todo el país tanto en la zona rural como la urbana. La población objetivo estuvo compuesta por los hogares particulares y sus miembros, de igual manera mujeres en edad de 15 a 49 años. La población oficialmente encuestada fue de 24 212, pero fueron seleccionados 17971 para el módulo de violencia doméstica. Considerándolos no encontrados, no respondieron y missing, realizamos nuestras regresiones con una muestra de 13667 datos válidos o consistentes.

\subsection{Determinantes de la violencia física}

Según la regresión econométrica efectuada, la probabilidad de que la mujer sufra agresión física por parte de su cónyuge es mayor en la medida que el hogar pertenezca a un estrato económico medio y pobre, la mujer esté desempleada, ella haya recibido maltrato físico familiar durante su infancia, esté en la condición de casada o de conviviente, haya alcanzado educación superior y cuando resida en la zona urbana y en la región de la costa. Los parámetros estimados correspondiente a estas variables explicativas son estadísticamente significativos (véase cuadro 1)

Cabe destacar la probabilidad de generación de violencia física en hogares de la clase media y pobre y, la relativa ausencia de ella en hogares de estratos económicos ricos o muy ricos. De la misma manera, el hecho de la mujer tenga mayor probabilidad de sufrir agresión física en la medida en que ella no esté incorporada al mercado laboral o que esté desempleada. 


\section{Juan León Mendoza}

Cuadro 1. Perú: Determinantes de la violencia física conyugal contra la mujer

\begin{tabular}{|l|c|c|}
\hline Variables independientes & $\mathbf{d y} / \mathbf{d x}$ & $\mathbf{P}>\mathbf{z}$ \\
\hline Familia de estrato pobre & 0.0406 & $0.002^{* *}$ \\
\hline Familia de estrato medio & 0.0488 & $0.003^{* *}$ \\
\hline Familia de estrato rico & 0.0289 & 0.130 \\
\hline Familia de estrato muy rico & -0.0166 & 0.449 \\
\hline Mujer en situación de desempleo & 0.0768 & $0.000^{* *}$ \\
\hline Agresión física paternal sufrida durante la niñez & 0.1059 & $0.000^{* *}$ \\
\hline Estado civil de casada o de conviviente & 0.1851 & $0.000^{* *}$ \\
\hline Edad de la mujerc & 0.0157 & $0.000^{* *}$ \\
\hline Edad de la mujer ${ }^{2 c}$ & -0.0001 & $0.003^{* *}$ \\
\hline Mujer de mediana calificación laboral & 0.0506 & $0.006^{* *}$ \\
\hline Mujer de baja calificación laboral & 0.0311 & 0.104 \\
\hline Educación primaria alcanzada por la mujer & 0.0259 & 0.217 \\
\hline Educación secundaria alcanzada por la mujer & 0.0366 & 0.099 \\
\hline Educación superior alcanzada por la mujer & -0.0736 & $0.002^{* *}$ \\
\hline Residencia en la zona urbana & 0.0277 & $0.019^{*}$ \\
\hline Residencia en la región costa & 0.0249 & $0.000^{* *}$ \\
\hline
\end{tabular}

Fuente: Regresión efectuada

$\mathrm{dy} / \mathrm{dx}$ is for discrete change of dummy variable from 0 to 1

(*) al $5 \%$ de error, $(* *)$ al $1 \%$ de error

Cuando el hogar es pobre y la mujer no está empleada en el mercado laboral, la probabilidad de violencia es mayor porque se incrementa el grado de dominio o poder económico del varón sobre su pareja. En contraposición, ello quiere decir que, si la mujer labora en el mercado de trabajo y coadyuva a que la familia goce de mayor estatus económico, tiende a ausentarse la probabilidad de violencia física conyugal.

La agresión física paternal atravesada por la mujer durante su niñez también coadyuva a la generación de la violencia física conyugal. En este caso, la agresión física paternal genera patologías de agresividad y predisposición a la violencia, lo cual al final se traduce en mayor ocurrencia de este fenómeno. 


\section{Pensamiento Crítico N. 15}

El hecho de que se tenga una mayor probabilidad de la violencia física en parejas casadas o convivientes y no en las separadas o divorciadas es esperable. En el marco de un sistema machista y patriarcal, en el caso de la primera situación, el varón tiene un control directo y mayor sobre su pareja, lo cual posibilita de alguna manera a una mayor ocurrencia de la agresión física.

La generación de la violencia cuando la mujer que trabaja es una mano de obra de mediana calificación, que cuenta con educación superior y radica en la zona urbana y región de la costa, estaría reflejando la respuesta agresiva del varón ante la relativa pérdida de control machista y patriarcal o ante la ganancia de empoderamiento de la mujer. Ello refleja que el proceso de empoderamiento de la mujer peruana está sujeto a ciertas resistencias de las parejas machistas y patriarcales, tal como lo señala también de alguna manera el estudio de Matos y Sulmont (2009).

\subsection{Determinantes de la violencia psicológica}

El Cuadro 2 muestra que la probabilidad de que la mujer sufra agresión psicológica por parte de su pareja es mayor en la medida en que el hogar pertenezca a un estrato socioeconómico pobre y medio, pero también se da en el estrato rico, asimismo, es mayor la probabilidad de maltrato cuando la mujer no trabaja en el mercado laboral, ha sufrido agresión física paternal durante su infancia, esté en situación de casada o conviviente, tenga hijos menores de cinco años, solo haya alcanzado educación primaria y secundaria y, cuando radique en la zona urbana y región de la costa.

A diferencia de la violencia física, en el caso de la violencia psicológica, la pertenencia del hogar a un estrato socioeconómico rico también se convierte en una de las variables condicionantes de la generación de la citada violencia. Este hecho refleja que en cada estrato económico los tipos de violencia que predominan pueden ser diferentes.

Si bien en el caso de la violencia física la probabilidad de que se genere la violencia conyugal contra la mujer es mayor cuando ella tiene educación superior, en el caso de la violencia psicológica dicha variable no tiene presencia explicativa; más bien, el hecho de que la mujer tenga sólo educación primaria y secundaria posibilita a que se produzcan actos de violencia psicológica. 


\section{Juan León Mendoza}

Cuadro 2. Perú: Determinantes de la violencia psicológica conyugal contra la mujer

\begin{tabular}{|l|c|r|}
\hline Variables independiente & $\mathbf{d y} / \mathbf{d x}$ & \multicolumn{1}{|c|}{$\mathbf{P}>\mathbf{z}$} \\
\hline Familia de estrato pobre & 0.0650 & $0.000^{* *}$ \\
\hline Familia de estrato medio & 0.0544 & $0.001^{* *}$ \\
\hline Familia de estrato rico & 0.0398 & $0.033^{*}$ \\
\hline Familia de estrato muy rico & -0.0083 & 0.692 \\
\hline Mujer en situación de desempleo & 0.0654 & $0.000^{* *}$ \\
\hline Agresión física paternal sufrida durante la niñez & 0.0886 & $0.000^{* *}$ \\
\hline Estado civil de casada o de conviviente & 0.2909 & $0.000^{* *}$ \\
\hline Tenencia de hijos menores de cinco años & 0.0127 & $0.026^{*}$ \\
\hline Edad de la mujerc & 0.0178 & $0.000^{* *}$ \\
\hline Edad de la mujer ${ }^{2 c}$ & -0.0001 & $0.001^{* *}$ \\
\hline Educación primaria alcanzada por la mujer & 0.0438 & $0.034^{*}$ \\
\hline Educación secundaria alcanzada por la mujer & 0.0605 & $0.006^{* *}$ \\
\hline Educación superior alcanzada por la mujer & -0.0229 & 0.326 \\
\hline Residencia en la zona urbana & 0.0355 & $0.002^{* *}$ \\
\hline Residencia en la región costa & 0.0395 & $0.000^{* *}$ \\
\hline
\end{tabular}

Fuente: Regresión efectuada

$\mathrm{dy} / \mathrm{dx}$ is for discrete change of dummy variable from 0 to 1

(*) al $5 \%$ de error, $(* *)$ al $1 \%$ de error

Finalmente, se debe subrayar que, la probabilidad de ocurrencia de la violencia tanto física como la psicológica están condicionados por el hecho de que el hogar pertenezca al estrato económico medio o pobre, que la mujer esté desempleada, que haya sufrido agresión física paternal en su infancia y esté en situación de casada o conviviente. 


\section{Pensamiento Crítico N. 15}

\subsection{Determinantes de la violencia sexual}

Según los resultados de la regresión, la probabilidad de que la mujer sufra violencia sexual por parte de su pareja es mayor en la medida en que ella no trabaje en el mercado laboral, haya recibido maltrato físico paternal durante su niñez, esté en situación de casada o de convivencia, cuando posea educación superior universitaria y resida en la región de la sierra (véase Cuadro 3).

A diferencia de la violencia física y psicológica, en el caso de la probabilidad de ocurrencia de la violencia sexual, el estrato social a la que pertenece la familia no tiene relevancia explicativa. También, en este caso de la violencia sexual, el hecho de que la mujer resida en la región de la sierra se convierte en una variable explicativa, aspecto que no se da en el caso de la violencia física y psicológica.

Cuadro 3. Perú: Determinantes de la violencia sexual conyugal contra la mujer

\begin{tabular}{|l|c|r|}
\hline Variable independiente & $\mathbf{d y} / \mathbf{d x}$ & \multicolumn{1}{|c|}{$\mathbf{P > \mathbf { z }}$} \\
\hline Mujer en situación de desempleo & 0.0229 & $0.000^{* *}$ \\
\hline Agresión física paternal sufrida durante la niñez & 0.0297 & $0.001^{* *}$ \\
\hline Estado civil de casada o de conviviente & 0.1330 & $0.000^{* *}$ \\
\hline Tenencia de hijos menores de cinco años & 0.0059 & 0.078 \\
\hline Edad de la mujerec & 0.0026 & $0.000^{* *}$ \\
\hline Educación primaria alcanzada por la mujer & 0.0027 & 0.880 \\
\hline Educación secundaria alcanzada por la mujer & -0.0082 & 0.651 \\
\hline Educación superior alcanzada por la mujer & -0.0372 & $0.009 * *$ \\
\hline Residencia en la zona urbana & 0.0105 & 0.063 \\
\hline Residencia en la región de la sierra & 0.0372 & $0.000^{* *}$ \\
\hline Residencia en la región de la selva & 0.0138 & 0.052 \\
\hline
\end{tabular}

Fuente: Regresión efectuada $\mathrm{dy} / \mathrm{dx}$ is for discrete change of dummy variable from 0 to 1

$(* *)$ al $1 \%$ de error 


\section{Juan León Mendoza}

\section{Conclusiones}

a) Dada la existencia de tres tipos de violencia contra las mujeres: física, psicológica y sexual, las variables explicativas no necesariamente son las mismas para cada una de ellas.

b) Las únicas variables que explican la probabilidad de ocurrencia tanto de la violencia física como de la violencia psicológica y sexual contra las parejas femeninas son: el que la mujer no cuente con empleo, que haya sufrido agresión física por parte de sus padres durante su niñez y, cuando ella esté casada o sea conviviente con su pareja.

\section{Bibliografía}

ACKERSON, LK, I. Kawachi, EM Barbeau, SV Subramanian (2008). Effects of individual and proximate educational context on intimate partner violence: a population- based study of women in India, Am. J Public Health,; 98: 507-14.

CASTRO, Roberto y Florinda Riquer (2003). La investigación sobre violencia contra las mujeres en América Latina: entre el empirismo ciego y la teoría sin datos, Cad. Saúde Pública, Rio de Janeiro, 19(1):135-146, jan-fev, 2003.

GONZALES De Olarte E. y P. Gavilano (1999). "¿Es la pobreza una causa de la violencia? Respuestas de Lima”. En El Costo Del Silencio: violencia doméstica en las América. Morrison, A. y Loreto, M. (editores), Washington: BID.

INSTITUTO NACIONAL DE ESTADÍSTICA E INFORMÁTICA (2010). Encuesta Demográfica y de Salud Familiar 2009. (ENDES 2009). Lima: INEI.

KNAUL, Felicia, Miguel Ángel Ramírez (1999). El impacto de la violencia intrafamiliar en la probabilidad de violencia intergeneracional, la progresión escolar y el mercado laboral en México, INEGI en la ciudad de México.

MATOS, Sylvia y SULMONT, David (2009). Modelos multivariados para la violencia conyugal, sus consecuencias y la solicitud de ayuda. Lima: Instituto Nacional de Estadística e Informática. 


\section{Pensamiento Crítico N. ${ }^{\circ}$}

OCHOA, Silvia (2002). Factores asociados a la presencia de violencia hacia la mujer. Lima: Instituto Nacional de Estadística e informática.

RUIZ-Pérez, I, J Plazaola-Castaño, M Álvarez-Kindelan, M Palomo-Pinto, M ArnalteBarrera, A Bonet-Pla et al.(2006). Sociodemographic association's o fphysical, emotional and sexual intimate partner violence in Spanis hwomen. Ann Epidemiol, 2006; 16: 357-63. 